\section{Recurrencias de malaria por \\ Plasmodium vivax según el \\ uso de primaquina. Análisis \\ de estudios descriptivos \\ longitudinales}

\section{Plasmodium vivax malaria \\ recurrence according to the use of \\ primaquine. Analysis of longitudinal \\ descriptive studies}

\section{Resumen}

Antecedentes: la primaquina (PQ) es el único medicamento disponible en el mercado para prevenir recurrencias del paludismo por Plasmodium vivax pero varios aspectos suyos se desconocen. Objetivo: comparar regímenes de PQ para prevenir recurrencias de malaria vivax. Metodología: revisión sistemática de datos. Resultados: 1 . ¿ Según los estudios descriptivos, la PQ es eficaz para prevenir las recurrencias del paludismo vivax? Sí. La comparación de estudios que no usaron PQ con otros que sí la aplicaron, en cualquier esquema, mostró que si no se usa PQ la recurrencia es altamente probable. 2. ¿Tienen la misma eficacia dosis diarias (mg/ $\mathrm{kg}$ ) iguales pero dosis totales diferentes? $\mathrm{La}$ dosis total de $75 \mathrm{mg}$ es tanto o más eficaz que la de $210 \mathrm{mg}$. 3. ¿La eficacia anti-recurrencias depende del lugar donde sucede la infección? Si. Hay variación según país y región. 4. ¿La frecuencia de recurrencias depende del tiempo de seguimiento post tratamiento? La respuesta no es uniforme para todos los lugares. Conclusiones: la PQ resultó eficaz para prevenir las recurrencias, pero no fue $100 \%$. Las dosis totales de $210 \mathrm{y}$ de $75 \mathrm{mg}$ tuvieron igual eficacia, pero $75 \mathrm{mg}$ sólo han sido evaluados en India, donde $P$. vivax parece ser más sensible a la $P Q$ que en otros lugares. Parece indudable la influencia del lugar en la proporción de recurrencias, incluso con una misma dosis total. El papel del tiempo de seguimiento no resultó claro. Deben evaluarse esquemas alternativos al estándar, que tiene eficacia promedio de $90 \%$ o más.

Palabras clave: Primaquina. Malaria. Plasmodium vivax. Recurrencia. Eficacia. Estudios descriptivos.

\section{Jaime Carmona-Fonseca}

"Grupo Salud y Comunidad César Uribe Piedrahíta" (Universidad de Antioquia)
Financiación: Se obtuvo financiación de: Colciencias (Código: 111549326134; Contrato 611-2009); Estrategia de Financiación Codi-Universidad de Antioquia 2011-2012; y Universidad de Antioquia. Declaración de conflictos: Ninguno para manifestar.

Correspondencia: Jaime Carmona-Fonseca. "Grupo Salud y Comunidad César Uribe Piedrahita", Universidad de Antioquia, Carrera 51D número 62-29 [Falta inserir CP], Medellín, Colombia. Mail: jaimecarmonaf@hotmail.com 


\section{Abstract}

Background: primaquine (PQ) is the only drug available in the market to prevent Plasmodium vivax malaria recurrence, but several aspects are still unknown. Objective: To compare PQ regimens to prevent recurrence of vivax malaria. Methods: systematic review and metaanalysis of data. Results: 1 . According to descriptive studies, is PQ effective in preventing recurrence of vivax malaria? Yes. The comparison of studies that did not use PQ to others that did, using any regimen, showed that if PQ is not used, recurrence is highly likely. 2. Are equal daily doses effective $(\mathrm{mg} / \mathrm{kg}$ ) but total doses different? The total dose of $75 \mathrm{mg}$ is equally or more effective than $210 \mathrm{mg}$. 3. Does the efficacy depend on where the infection happens? Yes. There is variation by country and region. 4. Does the recurrence rate depend on the post-treatment time follow-up? The answer is not uniform everywhere. Conclusions: Although not $100 \%, P Q$ is effective in preventing recurrence. Total doses of 210 and $75 \mathrm{mg}$ are equally effective, but $75 \mathrm{mg}$ alone has been evaluated in India, where P. vivax seems to respond better to $\mathrm{PQ}$ than elsewhere. The effect of place in the proportion of recurrences seems evident, even using the same total dose. The role of follow-up time is not clear. Although the standard regimen has an average effectiveness of $90 \%$ or more, alternative regimens should be assessed.

Keywords: Primaquine. Malaria. Plasmodium vivax. Recurrence. Efficacy. Descriptive studies.

\section{Introducción}

Plasmodium vivax es frecuente en Asia y en América (desde el norte de México hasta el norte de Argentina) y está casi ausente en África ${ }^{1}$. La reaparición o recurrencia de la parasitemia, con o sin síntomas asociados, semanas después de haber habido una curación de la enfermedad, con eliminación total de los hemoparásitos y de los síntomas, es un rasgo exclusivo del paludismo por $P$. vivax y $P$. ovale ${ }^{2-4}$.

La primaquina (PQ), una 8-aminoquinolina ${ }^{5}$, es más eficaz cuando se da simultáneamente con los esquizonticidas sanguíneos ${ }^{6} \mathrm{y}$ posee el espectro de actividad más amplio entre todos los antimaláricos ${ }^{7}$. En malaria vivax, el riesgo de recurrencia/ recaída cuando el tratamiento se hace únicamente con PQ es de $79-80 \%$, con 12 meses de seguimiento, dando tanto 15 como 22,5 mg mg/día por 14 días ${ }^{6,8}$, algo similar a tratarla solo con cloroquina como esquizonticoda y sin dar PQ, pues la recurrencia/ recaída fue de $99-100 \%{ }^{6}$, valor éste que los datos globales sitúan en $5 \%$ a $80 \%$ y más, dependiendo de la zona geográfica ${ }^{9}$. Ni el esquizonticida, cualquiera que sea, solo ni la PQ sola son capaces de evitar la recurrencia/ recaída, como ya se ha señalado ${ }^{9-10}$.

Los estudios iniciales hallaron que una dosis total (DT) de PQ de $200 \mathrm{mg}$ base produjo la curación y que, para un adulto, una dosis diaria de $15 \mathrm{mg}(0,25 \mathrm{mg} / \mathrm{kg} /$ día $)$ por 14 días fue bien tolerada; ese esquema se adoptó como el estándar anti-recurrencias ${ }^{9}$, inclusive por la Organización Mundial de la Salud $(\mathrm{OMS})^{4}$. El bloqueo de la transmisión a anofeles al usar PQ se logra con la dosis usual, que causa completa pérdida de la transmisión en 4-20 horas ${ }^{11}$.

Es costumbre no dar más de $210 \mathrm{mg}$ de DT de PQ, aunque el peso corporal sea mayor de 60 kg; así, aquellos con más de $60 \mathrm{~kg}$ absorben dosis menores de las que debieran tomar; esto parece contribuir a fallas terapéuticas de la $\mathrm{PQ}^{12}$.

Con la DT estándar de PQ (DTE-210-14: resultante de $0,25 \mathrm{mg} / \mathrm{kd} /$ día por 14 días) se obtiene una proporción de recurrencias de 
$26,7 \%(16 / 60)^{13}$. Una dosis única semanal de 30, 45 ó 60 mg dada por 8 semanas consecutivas (DT: 240, 360 y $480 \mathrm{mg}$ ) es bien tolerada y permite recurrencias en 56,10 y $6 \%$ de los casos, respectivamente, mostrando clara relación dosis-respuesta ${ }^{13}$. La dosis de 45 $\mathrm{mg} / \mathrm{semana}$ por 8 semanas es tan eficaz para evitar recurrencias como $30 \mathrm{mg} /$ día por 14 días (DT: $420 \mathrm{mg}$ ) o como $60 \mathrm{mg} /$ día por 7 días (DT: $420 \mathrm{mg})^{14}$.

A partir de la malaria en monos con P. cynomolgi y los estudios humanos (experimentales y de campo) con malaria por $P$. vivax, de hace más de 50 años, se piensa que la curación radical (curación clínica y parasitaria que incluye evitar las recaídas) de la infección con $P$. cynomolgi y con P. vivax es una función de la DT de 8-aminoquinolinas ${ }^{13,15}$.

La tolerancia de $P$. vivax a PQ sucede en el sureste de Asia y en Oceanía, pero el riesgo de falla terapéutica ha sido rara vez bien documentado en cualquier lugar ${ }^{9,16}$. La resistencia a la PQ por parte de los estadios sanguíneos de $P$. vivax existe ${ }^{17}$ pero es de poca importancia clínica y la que interesa para las recaídas es la que puedan presentan las formas tisulares hepáticas o hipnozoítos.

La revisión Cochrane 2009, con estudios clínicos controlados aleatorios, halló que ${ }^{18}$ 210-14 produjo significativamente menos recurrencias que el tratamiento únicamente con cloroquina, sin agregar PQ; 75-5 careció de efecto anti-recurrencias comparada con el tratamiento de solo cloroquina y entre 210-14 y 75-5 fue significativamente mejor 210-14. Esta revisión no dejó dudas sobre la eficacia y la mejor forma de usar la PQ, pero persisten dudas sobre la mejor dosificación, entendida esta en función de la dosis (mg/ $\mathrm{kg}$ ) (idebe mantenerse $0,25 \mathrm{mg} / \mathrm{kg} /$ día o deben ser más de 0,25 ?), del número de días en que la DT debe darse (ies igual $0,25 \mathrm{mg} /$ $\mathrm{kg} /$ día por 14 días que $0,50 \mathrm{mg} / \mathrm{kg} /$ día por 7 días, o que $\approx 1,25 \mathrm{mg} / \mathrm{kg} /$ día por 3 días $\mathrm{u}$ otra forma?) y idebe mantenerse el límite máximo de $210 \mathrm{mg}$ de DT o debe darse la cantidad que corresponda al peso corporal real? Los escasos datos disponibles indican que, al parecer, la DT $210 \mathrm{mg}$ no debe darse en tan pocos días como 3 ó 5 porque la frecuencia de recaídas se eleva muchísimo ${ }^{19}$, pero hay que evaluar más su aplicación en 7 días $^{19}$.

El objetivo de la presente revisión fue medir, mediante un análisis estadístico de estudios descriptivos longitudinales, la eficacia de la PQ para evitar las recurrencias de la malaria por $P$. vivax. El metanálisis tiene empleo más generalizado para evaluar ensayos clínicos aleatorios y puede aplicarse también en estudios descriptivos y de evaluación de pruebas diagnósticas y pronósticas $^{20}$.

\section{Materiales y métodos}

\section{Clase de estudio y preguntas específicas para resolver con el análisis}

Se aplicaron metodologías sugeridas para la revisión sistemática y el metanálisis $^{20-22}$. Se buscó dar respuesta a estas preguntas: $1-i \mathrm{La}$ PQ es eficaz para prevenir las recurrencias del paludismo vivax? 2- $i$ Tienen la misma eficacia dosis diarias $(\mathrm{mg} / \mathrm{kg})$ iguales pero DT diferentes? Se quiere comparar la eficacia de 0,25 mg/ día por 5 días con la misma dosis por 7 y 14 días, o sea comparar DT de 75, 105 y 210 mg. $3-$ ¿La eficacia de la PQ para prevenir las recurrencias del paludismo vivax depende del lugar donde sucede la infección? 4-iLa frecuencia de recurrencias depende del tiempo de seguimiento (número de meses) post tratamiento?

\section{Fuentes de información, palabras claves, fechas, idiomas}

Se buscó información en Lilacs, Scielo, PubMed (Medline), Cochrane, Embase, Free Medical Journals e Intermedicina. Se usó la clave "primaquina" (primaquine) combinada con una o varias más: "recurrencia" (recurrence), "recaída" (relapse), "vivax" (vivax). Se hizo búsqueda de información hasta el 10 de mayo de 2011. La búsqueda se hizo sin límite de idioma y de fecha. 


\section{Tipos de estudios y de participantes}

Se buscaron informes exclusivamente con diseño descriptivo longitudinal o estudio de una única cohorte o estudio de observación. Son estudios que aplicaron o no aplicaron PQ a pacientes para tratar la malaria vivax que padecían. Se trabajó con un grupo que, después de curado el ataque agudo - mediante el uso de esquizonticida, generalmente la cloroquina-, sus integrantes fueron seguidos por determinado tiempo con el fin de constatar si aparecía la recurrencia. El estudio debió medir la eficacia de cualquier régimen de administración de PQ, así no hubiese tenido como objetivo principal evaluar la eficacia anti-recurrencias de la PQ. Se excluyeron escritos sobre "presentación de caso" y estudios clínicos controlados. No se buscó información con autores ni con investigadores.

Se aceptaron estudios con adultos o niños, hombres o mujeres (excepto embarazadas). No se aceptaron pacientes con infecciones mixtas, sino exclusivamente por P. vivax. No se exigió exclusión de pacientes con deficiencia de glucosa 6 -fosfato deshidrogenasa G6PD.

Se incluyeron estudios que usaron cualquier esquizonticida (cloroquina, amodiaquina, artesunato, etc.), bien fuese solo o con PQ en cualquier esquema. Se exigió un seguimiento mínimo de los pacientes durante dos meses, de los cuales los primeros 28-30 días debieron corresponder a un estado de carencia de parasitemia asexual y de síntomas-signos clínicos, como expresión de que el ataque agudo había sido curado. Lo importante para resaltar es que los pacientes incluidos siempre se curaron del ataque agudo, que es acción principalmente del esquizonticida, y únicamente entonces, tras completar 28-30 días de curación, se sometieron a seguimiento para estudiar la recurrencia; ese seguimiento posterior fue variable.

\section{Información exigida a los artículos; evaluación de los informes}

- Describir con precisión el grupo de tra- tamiento;

- indicar el tamaño del grupo;

- indicar precisamente la dosis de PQ aplicada;

- indicar el tiempo de seguimiento (no se exigió un tiempo mínimo de seguimiento);

- indicar el número o la proporción de recurrencias en cada grupo;

- indicar con claridad y precisión si los sujetos estuvieron en área de transmisión malárica luego de recibir el tratamiento preventivo;

- manifestar explícitamente que el diagnóstico de infección palúdica se estableció con gota gruesa o con PCR, con inequívoca indicación de la especie de Plasmodium, sin importar la parasitemia hallada ni la presencia o ausencia de manifestaciones clínicas.

La calidad de los estudios incluidos se evaluó pero no fue criterio para excluirlos; así, por ejemplo, la omisión de los datos sobre pérdidas en el seguimiento para detectar infecciones no llevó a excluir esos informes; tampoco fueron criterio de exclusión la forma de asignación al grupo de tratamiento (aleatoria, no aleatoria), la omisión del dato sobre supervisión vs. no supervisión del tratamiento, la deficiencia del análisis estadístico, la magnitud de la eficacia del tratamiento para prevenir la malaria.

La búsqueda de informes, la verificación de los criterios de inclusión y la evaluación de cada artículo fueron ejecutadas directa y exclusivamente por el autor de este trabajo.

\section{Análisis estadístico}

De cada estudio se tomaron los datos sobre el tamaño de cada grupo de tratamiento evaluado, el número de recurrencias encontrado o la proporción de las mismas. La incidencia de recurrencias se calculó según el análisis por protocolo (recurrencias al final del seguimiento dividido por el número de personas que llegaron al final del seguimiento $)^{23-24}$. El tiempo de seguimiento para países y regiones fue ponderado para 
calcular el promedio y el factor de ponderación fue el número de pacientes en cada estudio: el número de meses de seguimiento se multiplicó por el número de personas seguidas; la sumatoria de esos productos se dividió por la suma de pacientes. El tiempo promedio de seguimiento, en meses, en cada país o región se calculó en función de todos los tratamientos evaluados en cada país o región.

Para grupos de estudios, según determinada variable (lugar, dosis de PQ, etc.), se calcularon el riesgo relativo (RR) y su intervalo de confianza del 95\% (IC95\%), con Epilnfo 6.04.

La distribución de frecuencias de la incidencia de malaria entre grupos según determinada variable (lugar, dosis de PQ, etc.) se analizó mediante una prueba ji (chi) cuadrada (X2) de Mantel-Hanszel (X2M-H) por medio de EpiInfo 6.04.

Siempre se consideró como estadísticamente significativa una probabilidad (p) menor de 0.05 , teniendo en cuenta los grados de libertad (gl) asociados.

\section{Resultados}

Se incluyeron 41 estudios descriptivos longitudinales de campo, en personas sin o con tratamiento con $\mathrm{PQ}$, contenidos en 33 referencias (Tabla 1-A) ${ }^{12,19,25-57}$. Fueron exclusivamente estudios descriptivos, con o sin asignación aleatoria, realizados entre 1945 y 2011, en diferentes países, tanto en zonas endémicas de malaria como libres de ella. Se hallaron cuatro esquemas de dosificación de la PQ:

- DTE-210-14 (el esquema más común, usado en Asia - excepto India- y en América);

- DT-210-7 (0,50 mg/kg/día por 7 días, usado en Brasil);

- DT-75-5 (0,25 mg/kg/día por 5 días, casi exclusivo de India);

- no administrar PQ.

De zonas no endémicas se hallaron 6 informes y de las endémicas se encontraron 35 (sureste de Asia: 11, India: 14, Pakistán: 2 ,
Etiopía: 1, América del Sur: 7). Los estudios incluidos abarcan a 24.143 pacientes, de los cuales 14.835 (62\%) recibieron PQ y 9.308 (38\%) no la usaron. En un mismo país hay áreas endémicas y no endémicas; los lugares más apropiados para los estudios sobre verdaderas recaídas son los que se hacen en zonas no endémicas, donde todas las recurrencias son verdaderas recaídas.

El tiempo de seguimiento de los pacientes varió intensamente: desde 2 hasta 60 meses, con promedio ponderado de 11,56 meses (zona no endémica: 6,82; zona endémica: 14,12). También varió fuertemente según la dosis de PQ: 210-14 tuvo 5,90 meses, valor cercano al de 210-7, que presentó 4,82 meses, pero estos dos fueron muy distantes de los otros dos: 75-5 mostró 15,56 meses; sin PQ apareció con 14,41 meses (Tabla 1-B).

La incidencia de recurrencias fue de 8,16 x 100 con 210-14; de 25,10 x 100 con 210-7; de 8,14 x 100 con 75-5; de 26,15 x 100 sin PQ (para cuatro proporciones $\mathrm{p}=0,000001$ ), pero no existe diferencia significativa entre 210-14 vs. $75-5$ ( $p>0,962$ ) ni entre 210-7 vs. 0 ( $p>0,701$ ) (Tabla 2-A). Esos hallazgos se ratifican con el análisis del riesgo relativo (RR) por regiones, en función de los esquemas de PQ (Tabla 3). Siempre, 210-14 comparada con no uso de PQ (0 mg) demostró un RR "protector" (menor de 1: a menor valor del $\mathrm{RR}$, mayor la protección). En la zona de endemia se obtuvo:

- muy elevada protección con 210-14, comparada con $0 \mathrm{mg}$ de PQ $(\mathrm{RR}=$ 0,09166);

- la eficacia de 210-7, comparada con 0 mg de PQ, es muy débil ( $R R=0,81559$ en Suramérica);

- la dosis 75-5, frente a $0 \mathrm{mg}$ de PQ, tiene clara eficacia $(\mathrm{RR}=0,31117$; India $\mathrm{RR}=$ 0,43858);

- la enorme ventaja de $210-14$ sobre $210-7$ y $75-5$ es clara: RR de 0,09466 y 0,29374 , respectivamente.

Un mismo tratamiento tuvo notoria variación en las recurrencias en función del lugar. El esquema 210-14 presentó 2,04 x 100 en el sureste de Asia, contra 10,98 en 
Tabla 1 - Incidencia de recurrencias observadas de malaria vivax según dosis total de primaquina en los estudios incluidos.

Table 1 - Incidence of vivax malaria recurrences observed according to total dose of primaquine in the studies included.

\begin{tabular}{|c|c|c|c|c|c|c|c|c|c|c|c|c|c|c|}
\hline \multicolumn{15}{|c|}{ A. Datos de cada estudio. / A. Data from each study. } \\
\hline \multirow{3}{*}{$\begin{array}{l}\text { Endemia } \\
\text { Lugar } \\
\text { No-Endemia }\end{array}$} & \multirow[t]{3}{*}{ meses } & \multicolumn{12}{|c|}{ Primaquina: dosis total (mg, días) } & \multirow[t]{3}{*}{ Autor-1; ref } \\
\hline & & \multicolumn{3}{|c|}{210 mg en 14 días } & \multicolumn{3}{|c|}{210 mg en 7 días } & \multicolumn{3}{|c|}{75 mg en 5 días } & \multicolumn{3}{|c|}{$0 \mathrm{mg}$} & \\
\hline & & nro $^{a}$ & $\mathrm{R}$ & no-R & nro & $\mathrm{R}$ & no-R & nro & $\mathrm{R}$ & no-R & nro & $\mathrm{R}$ & no-R & \\
\hline Brasil & 6 & 1.086 & 314 & 772 & & & & & & & & & & Boulos 1991; 30 \\
\hline Brasil & 6 & 50 & 7 & 43 & & & & & & & & & & Duarte $2011 ; 11$ \\
\hline New York & 7 & 60 & 26 & 34 & & & & & & & 15 & 7 & 8 & Smoak 1997; 52 \\
\hline Alemania & 18 & 56 & 7 & 49 & & & & & & & & & & Jelinek 1995; 39 \\
\hline Israel & 24 & 15 & 5 & 10 & & & & & & & & & & Schwartz 1998; 46 \\
\hline Colombo & 12 & & & & & & & 6 & 5 & 1 & & & & Fernandopulle 2003; 34 \\
\hline No-E & $6,82^{b}$ & 1.267 & 359 & 908 & & & & 6 & 5 & 1 & 15 & 7 & 8 & \\
\hline Endemia & & & & 0 & & & & & & & & & & \\
\hline Surcorea & 6 & & & 0 & & & & & & & 1.021 & 327 & 694 & e tres ref: $26-28$ \\
\hline Surcorea $^{c}$ & 6 & 2.375 & 57 & 2.318 & & & & & & & & & & Moon 2009; 43 \\
\hline Surcorea ${ }^{c}$ & 6 & 1.506 & 5 & 1.501 & & & & & & & & & & Moon 2009; 43 \\
\hline Tailandia & 2 & 43 & 2 & 41 & & & & & & & & & & Luxemburger 1999; 41 \\
\hline Tailandia & 1 & 53 & 3 & 50 & & & & & & & & & & Fryauff 1997; 35 \\
\hline Tailandia & 3 & 92 & 5 & 87 & & & & & & & & & & Maneeboonyang 2011; 42 \\
\hline Tailandia & 3 & 187 & 15 & 172 & & & & & & & & & & Takeuchi 2011; 55 \\
\hline Tailandia & 2 & & & & & & & & & & 342 & 143 & 199 & Luxemburger 1999; 41 \\
\hline Tailandia & 2 & & & & & & & & & & 536 & 63 & 473 & Awab 2010; 29 \\
\hline N. Guinea & 2 & & & & & & & & & & 54 & 54 & 0 & Fairley $1945 ; 33$ \\
\hline Pacífico & 3,5 & & & & & & & & & & 562 & 405 & 157 & Fairley $1945 ; 33$ \\
\hline Asia Este & 5,05 & 4.256 & 87 & 4.169 & & & & & & & 2.515 & 992 & 1.523 & \\
\hline India & 18 & & & & & & & 5.541 & 511 & 5.030 & & & & Dua $2001 ; 32$ \\
\hline India & 6 & & & & & & & 165 & 15 & 150 & & & & Gogtay 1998; 36 \\
\hline India & 18 & & & & & & & 725 & 50 & 675 & & & & Sinha junio 1989; 50 \\
\hline India & 12 & & & & & & & 100 & 6 & 94 & 200 & 56 & 144 & Srivastava 1996; 54 \\
\hline India & 12 & & & & & & & 1.520 & 40 & 1.480 & & & & Sharma 1990; 48 \\
\hline India & 8 & & & & & & & & & & 264 & 50 & 214 & Sharma 1990; 48 \\
\hline India & 8 & & & & & & & 995 & 104 & 891 & 2.500 & 222 & 2278 & Singh 1990; 49 \\
\hline India & 12 & & & & & & & & & & 283 & 19 & 264 & Gogtay 2000; 37 \\
\hline India & 60 & & & & & & & & & & 316 & 140 & 176 & Adak 1998; 25 \\
\hline India & 48 & & & & & & & & & & 487 & 147 & 340 & Adak 1998; 25 \\
\hline India & 36 & & & & & & & & & & 497 & 132 & 365 & Adak 1998; 25 \\
\hline India & 24 & & & & & & & & & & 524 & 149 & 375 & Adak 1998; 25 \\
\hline India & 12 & & & & & & & & & & 669 & 156 & 513 & Adak 1998; 25 \\
\hline India & 12 & & & & & & & & & & 222 & 20 & 202 & Sinha sep 1989; 51 \\
\hline India & $16,69^{d}$ & & & & & & & 9.046 & 726 & 8.320 & 5.962 & 1.091 & 4.871 & \\
\hline Pakistán & 12 & & & & & & & & & & 250 & 130 & 120 & Rowland 1999; 45 \\
\hline Pakistán & 6 & & & & & & & & & & 212 & 99 & 113 & Leslie 2004; 40 \\
\hline Etiopía & 3 & & & & & & & & & & 29 & 15 & 14 & Schwartz 1998; 46 \\
\hline Paki-Etiop & 8,88 & & & & & & & & & & 491 & 8,88 & 491 & \\
\hline Asia & 13 & 4.256 & 87 & 4.169 & & & & 9.046 & 726 & 8.320 & 8.968 & 2.327 & 6.641 & \\
\hline Brasil & 3 & 31 & 2 & 29 & & & & 30 & 8 & 22 & & & & Villalobos 2000; 56 \\
\hline Brasil & 4 & 37 & 2 & 35 & 36 & 0 & 36 & & & & & & & Abdon $2001 ; 24$ \\
\hline Brasil & 6 & & & & 164 & 55 & 109 & & & & & & & Orjuela 2009; 44 \\
\hline Perú & 2 & 28 & 2 & 26 & 28 & 3 & 25 & & & & & & & Solari 2002; 53 \\
\hline Colombia & 6 & 68 & 12 & 56 & & & & & & & & & & Carmona 2009-a; 31 \\
\hline Colombia & 4 & & & & 35 & 8 & 27 & & & & & & & Carmona 2009-b; 18 \\
\hline
\end{tabular}


Tabla 1 - Incidencia de recurrencias observadas de malaria vivax según dosis total de primaquina en los estudios incluidos. (cont.)

Table 1 - Incidence of vivax malaria recurrences observed according to total dose of primaquine in the studies included. (cont.)

\begin{tabular}{|c|c|c|c|c|c|c|c|c|c|c|c|c|c|c|}
\hline \multirow{3}{*}{$\begin{array}{l}\text { Endemia } \\
\text { Lugar } \\
\text { No-Endemia }\end{array}$} & \multirow[t]{3}{*}{ meses } & \multicolumn{12}{|c|}{ Primaquina: dosis total (mg, días) } & \multirow[t]{3}{*}{ Autor-1; ref } \\
\hline & & \multicolumn{3}{|c|}{210 mg en 14 días } & \multicolumn{3}{|c|}{210 mg en 7 días } & \multicolumn{3}{|c|}{75 mg en 5 días } & \multicolumn{3}{|c|}{$0 \mathrm{mg}$} & \\
\hline & & nro $^{a}$ & $\mathrm{R}$ & no-R & nro & $\mathrm{R}$ & no- $R$ & nro & $\mathrm{R}$ & no-R & nro & $\mathrm{R}$ & no-R & \\
\hline Guyana & 30 & & & & & & & & & & 325 & 100 & 225 & Hanf et $2009 ; 38$ \\
\hline \multicolumn{15}{|l|}{ Francesa } \\
\hline Suramérica & 15,1 & 164 & 18 & 146 & 263 & 66 & 197 & 30 & 8 & 22 & 325 & 100 & 225 & \\
\hline No endemia & 6,82 & 1.267 & 359 & 908 & & & & 6 & 5 & 1 & 15 & 7 & 8 & \\
\hline Endemia & 14,12 & 4.420 & 105 & 4.315 & 263 & 66 & 197 & 9.076 & 734 & 8.342 & 9.293 & 2.427 & 6.866 & \\
\hline General & 11,56 & 5.687 & 464 & 5.223 & 263 & 66 & 197 & 9.082 & 739 & 8.343 & 9.308 & 2.434 & 6.874 & \\
\hline
\end{tabular}

a nro: número de pacientes en cada tratamiento; $R$ recurrencia, no- $R$ no recurrencia.

nro: number of patients in each treatment, $R$ recurrence; no- $R$ non- recurrence.

b El tiempo promedio de seguimiento, en meses, en cada país o región, se calculó en función de todos los tratamientos evaluados en cada país o región. Ver abajo los tiempos ponderados de seguimiento para las regiones, en cada tratamiento.

The average follow-up time (months) in each country or region was calculated based on all treatments evaluated in each country or region. See below for follow-up time weighted regions in each treatment.

'Surcorea: 2.375 soldados, con re-exposición a malaria después del tratamiento; 1.506 veteranos, sin re-exposición a malaria después del tratamiento. En este último grupo las recurrencias son recaídas genuinas.

South Korea: 2,375 soldiers, with re-exposure to malaria after treatment; 1,506 veterans, without re-exposure to malaria after treatment. In the latter group relapsed recurrences are genuine.

${ }^{d}$ El seguimiento promedio para los dos esquemas evaluados en India fue de 16,97 meses. Para PQ 75-5, el seguimiento promedio fue de 15,61 meses; para PQ 0 mg, el seguimiento promedio fue de 19,13 meses.

The average follow-up for the two regimens in India was 16.97 months. For PQ 75-5, the average follow-up was 15.61 months; for PQ with 0 mg, mean follow-up was 19.13 months.

eAlving et al 1953 (26); Hankey et al 1953 (27); Coatney et al 1953 (28)

\section{B. Tiempos de seguimiento ponderados (meses) para las regiones, en cada tratamiento. B. Weighted follow-up time (months) for regions, in each treatment.}

Zona PQ: esquema de dosificación (mg totales-días de repartición)

\begin{tabular}{|c|c|c|c|c|c|c|c|c|c|c|}
\hline & \multirow{2}{*}{\multicolumn{2}{|c|}{$210-14$}} & \multirow{2}{*}{\multicolumn{2}{|c|}{$210-7$}} & & & & & & \\
\hline & & & & & \multicolumn{2}{|c|}{$75-5$} & \multicolumn{2}{|c|}{0} & \multicolumn{2}{|c|}{ Total } \\
\hline & Mes & nro & Mes & nro & Mes & nro & Mes & nro & Mes & Nro \\
\hline No-Endemia & 6,79 & 1.267 & $--^{a}$ & -- & 6 & 6 & 7 & 15 & 6,82 & 1.288 \\
\hline Endemia & 5,65 & 4.420 & 4,82 & 263 & 15,57 & 9.076 & 14,42 & 9.557 & 13,09 & 23.316 \\
\hline Asia & -- & -- & -- & -- & 15,61 & 9.046 & 13,87 & 9.232 & 13,02 & 22.534 \\
\hline Asia Este & 5,71 & 4.256 & 3,95 & 2.530 & -- & -- & 3,96 & 2.515 & 5,05 & 6.771 \\
\hline India & -- & -- & -- & -- & -- & 9.046 & 18,27 & 6.226 & 16,69 & 15.262 \\
\hline Pakistán-Etiopía & -- & -- & -- & -- & -- & -- & 8,88 & 491 & 8,88 & 491 \\
\hline Suramérica & 4,30 & 164 & 4,82 & 263 & 3 & 30 & 30 & 325 & 15,1 & 782 \\
\hline No-End+End & 5,90 & 5.687 & 4,82 & 263 & 15,56 & 9.082 & 14,41 & 9.572 & 12,76 & 24.604 \\
\hline Pacientes & & \multicolumn{5}{|c|}{ Con PQ $5.687+263+9.082=15.032$} & \multicolumn{2}{|c|}{$\operatorname{Sin} P Q=9.572$} & \multicolumn{2}{|c|}{ Total $=24.604$} \\
\hline
\end{tabular}

a No hubo estudios. / No studies.

b El tiempo promedio de seguimiento, en meses, en cada país o región, se calculó en función de todos los tratamientos evaluados en cada país o región.

The average follow-up time (months) in each country or region was calculated based on all treatments evaluated in each country or region.

Suramérica $(\mathrm{p}=0,000000)$. En una misma región y bajo un mismo esquema de PQ, hay fuerte variación en la incidencia de recurrencias por países (Tabla 2-B). En India únicamente se han evaluado las DT 0 y 75-5. El seguimiento ha variado entre 5 y 60 meses. Con 75-5 se evaluaron 9.082 pacientes, con recurrencias de $8,14 \%$; sin PQ se evaluaron 9.308 pacientes y recurrencias en $26,15 \%$ (p $=0,000000$ ), datos que indican que en India el uso de PQ protege en forma altamente significativa contra las recurrencias. En Corea del Sur, donde las recurrencias son mucho más bajas que en los demás países 
Tabla 2 - Incidencia $\left(x 10^{2}\right)$ de recurrencias de malaria vivax según dosis total de primaquina en cada estudio. Table 2 -Incidence $\left(x 10^{2}\right)$ of recurrent vivax malaria according to total dose of $P Q$ in each study.

A. Incidencia en cada estudio / A. Incidence in each study.

\begin{tabular}{|c|c|c|c|c|c|}
\hline \multirow{2}{*}{$\begin{array}{l}\text { Endemia y lugar } \\
\text { No-E }\end{array}$} & \multicolumn{4}{|c|}{ Incidencia ponderada de Recurrencias $\times 10^{2}$ por dosis total $\mathrm{PQ}$} & \multirow[t]{2}{*}{ Autor-1; ref } \\
\hline & $210-14$ & $210-7$ & $75-5$ & 0 & \\
\hline Brasil & 28,91 & & & & Boulos 1991; 30 \\
\hline Brasil & 14,00 & & & & Duarte 2011; 11 \\
\hline N. York & 43,33 & & & 46,67 & Smoak 1997; 52 \\
\hline Alemania & 12,50 & & & & Jelinek 1995; 39 \\
\hline Israel & 33,33 & & & & Schwartz 1998; 46 \\
\hline Colombo & & & 83,33 & & Fernandopulle 2003; 34 \\
\hline No-E & 28,33 & & 83,33 & 46,67 & \\
\hline \multicolumn{6}{|l|}{ Endemia } \\
\hline Surcorea & & & & 32,03 & e $26-28$ \\
\hline Surcorea & 2,40 & & & & Moon 2009; 43 \\
\hline Surcorea & 0,33 & & & & Moon 2009; 43 \\
\hline Tailandia & 4,65 & & & & Luxemburger 1999; 41 \\
\hline Tailandia & 5,66 & & & & Fryauff 1997; 35 \\
\hline Tailandia & 5,43 & & & & Maneeboonyang 2011; 42 \\
\hline Tailandia & 8,02 & & & & Takeuchi 2011; 55 \\
\hline Tailandia & & & & 41,81 & Luxemburger 1999; 41 \\
\hline Tailandia & & & & 11,75 & Awab 2010; 29 \\
\hline N. Guinea & & & & 100,00 & Fairley 1945; 33 \\
\hline Pacífico & & & & 72,06 & Fairley 1945; 33 \\
\hline Asia Este & 2,04 & & & 39,44 & \\
\hline India & & & 9,22 & & Dua \& Sharma 2001 \\
\hline India & & & 9,09 & & Gogtay et al. 1998 \\
\hline India & & & 6,90 & & Sinha et al. 1989 Jun \\
\hline India & & & 6,00 & 28,00 & Srivastava et al. 1996 \\
\hline India & & & 2,63 & 18,94 & Sharma et al. 1990 \\
\hline India & & & 10,45 & 8,88 & Singh et al. 1990 \\
\hline India & & & & 6,71 & Gogtay et al. 2000 \\
\hline India & & & & 44,30 & Adak et al 1998 \\
\hline India & & & & 30,18 & Adak et al 1998 \\
\hline India & & & & 26,56 & Adak et al 1998 \\
\hline India & & & & 28,44 & Adak et al 1998 \\
\hline India & & & & 23,32 & Adak et al 1998 \\
\hline India & & & & 9,01 & Sinha et al. 1989 Sep \\
\hline India & & & & 18,94 & Singh et al. 1990 \\
\hline India & & & 8,03 & 18,33 & \\
\hline Pakistán & & & & 52,00 & Rowland et al 1999 \\
\hline Pakistán & & & & 46,70 & Leslie et al 2004 \\
\hline Etiopía & & & & 51,72 & Schwartz \& Sidi 1998 \\
\hline Pak-Etio & & & & 49,69 & \\
\hline Asia & & & 8,03 & 25,75 & \\
\hline
\end{tabular}


Tabla 2 - Incidencia $\left(\times 10^{2}\right)$ de recurrencias de malaria vivax según dosis total de primaquina en cada estudio. (cont.) Table 2 - Incidence $\left(x 10^{2}\right)$ of recurrent vivax malaria according to total dose of $P Q$ in each study. (cont.)

\begin{tabular}{|c|c|c|c|c|c|}
\hline \multirow[t]{2}{*}{ Endemia y lugar } & \multicolumn{4}{|c|}{ Incidencia ponderada de Recurrencias $\times 10^{2}$ por dosis total PQ } & \multirow[t]{2}{*}{ Autor-1; ref } \\
\hline & $210-14$ & $210-7$ & $75-5$ & 0 & \\
\hline Brasil & 6,45 & & 26,67 & & Villalobos-Salcedo et al 2000 \\
\hline Brasil & & 0,00 & & & Abdon et al 2001 \\
\hline Brasil & & 33,54 & & & Orjuela et 2009 \\
\hline Perú & 7,14 & 10,71 & & & Solari-Soto et al 2002 \\
\hline Colombia & 17,65 & & & & Carmona et al 2009-a \\
\hline Colombia & & 22,86 & & & Carmona 2009-b \\
\hline Guyana Francesa & & & & 30,77 & Hanf et 2009 \\
\hline Suramérica & 10,98 & 25,10 & & 30,77 & \\
\hline No endemia & 28,33 & & 83,33 & 46,67 & \\
\hline Endemia & 2,38 & 25,10 & 8,09 & 26,12 & \\
\hline General & 8,16 & 25,10 & 8,14 & 26,15 & \\
\hline
\end{tabular}

\section{B. Resumen de la tabla 2-A / B. Summary of Table 2-A}

\begin{tabular}{lcccc}
\hline \multirow{2}{*}{ Endemia y lugar } & \multicolumn{3}{c}{ Incidencia de Recurrencias $\times 10^{2}$ por dosis total PQ } \\
\cline { 2 - 4 } $\begin{array}{l}\text { No-Endemia } \\
\text { Endemia }\end{array} \quad 210-14$ & $210-7$ & $75-5$ & 0 \\
$\quad$ Asia Este & 28,33 & 83,33 & 46,67 \\
$\quad$ India & 2,04 & & 39,44 \\
$\quad$ Pak-Etio & & 8,03 & 18,30 \\
$\quad$ Asia & & & 49,69 \\
$\quad$ Suramérica & 10,98 & & 8,03 & 25,95 \\
No endemia & $28,33(\mathrm{n}=1.267)^{\mathrm{a}}$ & & & 30,77 \\
Endemia & $2,38(\mathrm{n}=4.420)$ & $25,10(\mathrm{n}=263)$ & $8,09(\mathrm{n}=9.076)$ & $26,12(\mathrm{n}=9.293)$ \\
General & $8,16(\mathrm{n}=5.687)$ & $25,10(\mathrm{n}=263)$ & $8,14(\mathrm{n}=9.082)$ & $26,15(\mathrm{n}=9.308)$
\end{tabular}

${ }^{a} n$ : total de pacientes en cada esquema de $P Q / n$ : total number of patients in each $P Q$ regimen.

Para 4 grupos: $\mathrm{p}(\mathrm{X} 2)=0,000001 /$ For 4 groups: $p(X 2)=0.000001$.

Valores de $\mathrm{p}(\mathrm{X} 2)$ para las comparaciones de pares de esquemas de $\mathrm{PQ}$ :

Values of $p(X 2)$ for comparisons of pairs of $P Q$ regimens:

\begin{tabular}{lccc}
\hline \multirow{2}{*}{ Esquema; $\%$} & No-endemia & Endemia & General \\
\cline { 2 - 4 } & \multicolumn{3}{c}{ Incidencias; } \\
\hline $210-14$ vs 0 & 28,33 vs 46,67 & 2,38 vs 26,12 & 8,16 vs 26,15 \\
& 0,202217 & 0,000000 & 0,000000 \\
\hline $210-14$ vs $75-5$ & 28,33 vs 83,33 & 2,38 vs 8,09 & 8,16 vs 8,14 \\
& 0,011683 & 0,001020 & 0,962090 \\
\hline $210-14$ vs $210-7$ & n.a & 2,38 vs 25,10 & 8,16 vs 25,10 \\
& & 0,000000 & 0,000000 \\
\hline $210-7$ vs 0 & n.a & 25,10 vs 26,12 & 25,10 vs 26,12 \\
& & 0,763710 & 0,701053 \\
\hline $210-7$ vs $75-5$ & n.a & 25,10 vs 8,09 & 25,10 vs 8,14 \\
& & 0,000000 & 0,000000 \\
\hline $75-5$ vs 0 & 86,33 vs 46,67 & 8,09 vs 26,12 & 8,14 vs 26,15 \\
& 0,295642 & 0,000000 & 0,000000 \\
\hline
\end{tabular}


Tabla 3 - Riesgo relativo entre esquemas de primaquina, según regiones, para la incidencia de recurrencias de malaria vivax.

Table 3 - Relative risk among primaquine regimens, according to regions, for the incidence of vivax malaria recurrences.

\begin{tabular}{|c|c|c|c|c|c|c|c|}
\hline \multirow[b]{2}{*}{ Endemia y lugar } & \multirow{2}{*}{$\begin{array}{l}\text { Meses } \\
\text { Seguim/ }\end{array}$} & \multicolumn{6}{|c|}{ Riesgo Relativo a entre esquemas de PQ } \\
\hline & & $\begin{array}{c}210-14 \text { vs } \\
0\end{array}$ & $\begin{array}{c}210-7 \text { vs } \\
0 \\
\end{array}$ & $\begin{array}{c}75-5 \text { vs } \\
0\end{array}$ & $\begin{array}{c}210-14 \text { vs } \\
210-7\end{array}$ & $\begin{array}{c}210-14 \text { vs } \\
75-5\end{array}$ & $\begin{array}{c}210-7 \text { vs } \\
75-5\end{array}$ \\
\hline No-E & 6,82 & 0,60717 & & 1,78571 & 1,78571 & 0,34002 & \\
\hline Asia & 8,91 & & & 0,30930 & & & \\
\hline Asia Este & 5,05 & 0,05183 & & & & & \\
\hline India & 16,97 & & & 0,43858 & & & \\
\hline Suramérica & 14,60 & 0,35671 & 0,81559 & & 0,43736 & & \\
\hline No endemia & 6,81 & $\begin{array}{c}0,60717 \\
(0,35 a 1,05)^{b}\end{array}$ & & 1,78571 & & $\begin{array}{c}0,34002 \\
(0,24 a 0,49)\end{array}$ & \\
\hline Endemia & 14,12 & $\begin{array}{c}0,09096 \\
(0,08 a 0,11)\end{array}$ & 0,96089 & $\begin{array}{c}0,30966 \\
(0,29 a 0,34)\end{array}$ & $\begin{array}{c}0,09466 \\
(0,07 a 0,13)\end{array}$ & $\begin{array}{c}0,29374 \\
(0,24 a 0,36)\end{array}$ & 3,10303 \\
\hline General & 13,68 & $\begin{array}{c}0,31201 \\
(0,29 a 0,35)\end{array}$ & 0,95967 & $\begin{array}{c}0,31117 \\
(0,29 a 0,34)\end{array}$ & $\begin{array}{c}0,32512 \\
(0,26 a 041)\end{array}$ & 1,00270 & 3,08408 \\
\hline
\end{tabular}

a RR menor de 1 indica mayor protección por el tratamiento mencionado en primer lugar; RR mayor de 1 indica menor protección por el tratamiento mencionado en primer lugar.

$R R$ less than 1 indicates greater protection by the first mentioned treatment; RR greater than 1 indicates less protection by the first listed treatment.

b Intervalo de confianza de $95 \%$ para el RR. No se hizo el cálculo para regiones ni para los totales (endemia, no endemia, general) con RR cercano a 1.

95\%Confidence interval for RR. Not calculated for regions and for total (endemic, not endemic, general) with a RR close to 1.

endémicos analizados, se evaluaron 210-14 y no PQ: las recurrencias con 210-14 fueron $15,15 \%(\mathrm{n}=3.819)$ y $\sin \mathrm{PQ}$ fueron $32,03 \%(\mathrm{n}$ $=1.021)(\mathrm{p}=0,000000)$. En Suramérica, 21014 se asoció a una proporción de recurrencias de 10,98; por su parte, 210-7 produjo 25,10 y el no uso de PQ tuvo 30,77 x 100 . Es consistente el hallazgo de que la $P Q$ protege fuertemente contras las recurrencias, pero no hay relación dosis respuesta, pues 75 y $210 \mathrm{mg}$ de DT protegieron igual.

La influencia del lugar es clara también al analizar las recurrencias en pacientes sin PQ. En India hubo 18,33 \% (1.141/6.226) de recurrencias; en Tailandia fueron $23,46 \%$ (206/878); en Pakistán fueron 49,57\% (229/462) y en Guyana Francesa fueron $30,77 \%$. (100/325).

En resumen, el uso de PQ para prevenir las recurrencias mostró una fortísima influencia del lugar (país, región), aunque en general parece que puede decirse que la PQ previene las recurrencias comparada con no usarla, pero la diferencia entre usar 75 y 210 mg de DT no es clara.

El coeficiente de correlación lineal entre proporción de recurrencias y meses de seguimiento es 0,0337 para el total de estudios (sugiere, con fuerza, que el tiempo de seguimiento no aumenta las recurrencias). En Tailandia los estudios han seguido los pacientes por apenas 1-3 meses, con $210 \mathrm{mg}$ de PQ; para cuatro estudios ${ }^{14}$, el coeficiente de correlación es de 0,44445 (a mayor seguimiento, más recurrencias). En India, para seis estudios con dosis total de $75 \mathrm{mg}$, el coeficiente es - 0,20398 (a más seguimiento, menos recurrencias). En India, para once estudios sin PQ, el coeficiente es 0,79125 (sugiere, con fuerza, que a mayor seguimiento, más recurrencias). En otros países no es posible este análisis por la escasez de estudios.

En resumen, el tiempo de seguimiento, como lo hizo el lugar, también influye en la proporción de recurrencias, al menos en los estudios sin PQ o con 210 mg de DT.

\section{Las preguntas de estudio y sus respuestas}

- ¿La PQ es eficaz para prevenir las recurrencias del paludismo vivax? La 
respuesta es sí. La comparación de estudios que no usaron PQ con otros que sí la aplicaron, en cualquiera de los esquemas, mostró que si no se usa PQ la recurrencia es muy probable.

- 2. ¿Tienen la misma eficacia dosis diarias iguales pero DT diferentes? Se compararon 75-5 y 210-14. La respuesta no es clara; los datos sugieren que $75-5$ es tanto o más eficaz que 210-14.

- 3. ¿La eficacia de la PQ para prevenir las recurrencias del paludismo vivax depende del lugar donde sucede la infección? La respuesta es sí. Los datos indicaron variación de recurrencias según el país y la región.

- 4. ¿La frecuencia de recurrencias depende del tiempo de seguimiento (meses) post tratamiento? La respuesta no es uniforme para todos los lugares. En Tailandia y en India, con 210-14 y 0 mg de $P Q$, respectivamente, las recurrencias aumentan con el tiempo de seguimiento, pero en India, con 75-5, parecen reducirse.

\section{Discusión}

La pregunta sobre si, según los estudios descriptivos, la PQ es eficaz para prevenir las recurrencias del paludismo vivax, pudo responderse afirmativamente. Se demostró que la PQ en DTE-210-14 o en DT-75-5 redujo las recurrencias, sin relación dosis-respuesta.

La pregunta sobre si tienen la misma eficacia dosis diarias $(\mathrm{mg} / \mathrm{kg})$ iguales pero dosis totales (DT) diferentes (DT de 75, 105 y $210 \mathrm{mg}$ ) también se respondió afirmativamente. Contra lo esperado ${ }^{4}$, DT-75-5 en la India protegió de recurrencias tanto como DT-210-14 en otros lugares. Las recurrencias con DT-75-5 únicamente pudieron evaluarse en India. Urge evaluar esquemas de PQ que suministren $210 \mathrm{mg}$ en 7 días, pues los trabajos son pocos (Brasil ${ }^{25,57-59}$, un estudio en Perú ${ }^{54}$ y otro en Colombia ${ }^{19}$ ).

La pregunta sobre si la eficacia de la PQ para prevenir las recurrencias del paludismo vivax depende del lugar donde sucede la infección también tuvo respuesta afirmativa. En efecto, este análisis demostró que el lugar o geografía de la malaria vivax influye poderosamente en la respuesta a la PQ. Hay que recordar que existen diferencias regionales importantes en la eficacia antirecurrencias de la PQ. En Tailandia, el riesgo de recurrencias fue dos veces mayor que en Brasil y 10 veces mayor que en India ${ }^{60}$. Esto puede explicar el hecho de que en India 75-5 sea eficaz para prevenir las recurrencias en $80-90 \%$ de los pacientes ${ }^{61}$, lo cual reitera que $P$. vivax en India es particularmente susceptible a PQ.

La probabilidad de recurrencias sin tratamiento con PQ varía según la región geográfica ${ }^{9}$ : en regiones templadas las recaídas suceden a intervalos prolongados, alrededor de 16 meses $^{62}$, mientras que en zonas tropicales la infección está asociada con mayor probabilidad de recaídas, que suceden más rápidamente y son múltiples ${ }^{9}$.

Hay variación geográfica de la susceptibilidad de P. vivax a cloroquina y $\mathrm{PQ}$, como lo demostró una revisión de $2007^{60}$, al comparar los riesgos en Tailandia, Brasil e India. Comparado con 0 mg de PQ, el riesgo se redujo $80 \%$ para $210-14$ y más de 95\% para 315-14 y 420-14. En la presente revisión, también resultó muy notoria la influencia del lugar en la proporción de recurrencias con un mismo tratamiento con PQ. Conviene insistir un poco más en la variación geográfica de las recurrencias post tratamiento con PQ. El inmenso subcontinente de India, con sus 22 estados, es el séptimo país más extenso del mundo y tiene ahora más de 1,2 millones de habitantes. El clima varía desde tropical y monzónico en el sur del país a templado en el norte. Toda India es endémica de malaria, con excepción de las áreas con 2.000 y más metros de altura sobre el nivel del mar. La malaria es estable en el noreste e inestable en el resto de la península. La frecuencia de $P$. vivax varía entre 55 y $100 \%$ según el estado ${ }^{63}$. Las recurrencias no son uniformes sino que varían de $9 \%$ a $40 \%$ sin PQ, de $6,5 \%$ a $30 \%$ con PQ 75-5, y de 0 a $6 \%$ con PQ 210-14, es decir que 210-14 es francamente superior a 75-5 
para evitar las recurrencias. La frecuencia de recurrencias en algunos estados, luego del tratamiento con cloroquina y sin PQ y cloroquina con PQ es así: Delhi (al norte) $35 \%$ sin PQ y $27 \%$ con PQ; Gujarat (oeste): $28 \%$ y $6 \%$, en ese orden; Madhya Pradesh (centro): $9 \%$ y $10 \%$, en igual orden; Orissa (este): $9 \%$ y 7\%; Mumbai (oeste): $11 \% 3 \%$ (adaptado de ${ }^{63}$ ).

La pregunta sobre si la frecuencia de recurrencias depende del tiempo de seguimiento (número de meses) post tratamiento tuvo respuesta negativa en el total de estudios, pero afirmativa según la región, como en Tailandia e India, análisis que no fue posible para otros países por el reducido número de estudios. En resumen, el tiempo de seguimiento también influye en la proporción de recurrencias, al menos en los estudios sin PQ o con $210 \mathrm{mg}$ de DT.

Un problema adicional, no analizado en este trabajo, se refiere a la subdosificación de PQ por la costumbre de no dar más de $210 \mathrm{mg}$ de PQ; es posible que ello contribuya a fallas terapéuticas de la PQ. En Brasil, la DT de PQ inferior a lo requerido por el peso corporal está entre "factores de riesgo" asociados a recurrencias ${ }^{12,64} \mathrm{y}$ ha sido reiterado ${ }^{64}$. No hay análisis de fondo, en diversos lugares, sobre la repercusión real de esta subdosificación.

En esta revisión no se tuvo en cuenta la clase de esquizonticida usado para tratar el ataque primario de paludismo vivax. Los esquizonticidas pueden interactuar con la PQ y aumentar o reducir la acción de ella ${ }^{65-66}$. Lo fundamental para destacar aquí es que los pacientes incluidos siempre se curaron del ataque agudo, cualquiera haya sido el esquizonticida. Después de 24-25 días de curación, se pusieron en seguimiento para estudiar la recurrencia.

Se ha anotado que existe tolerancia de $P$. vivax a PQ (sureste de Asia y Oceanía) ${ }^{9,16}$ que la resistencia a PQ por estadios sanguíneos de P. vivax existe ${ }^{17}$, pero carece de importancia clínica. Las formas que importan para las recaídas son los estadios tisulares hepáticos o hipnozoítos, que no causan síntomassignos mientras están como tales, como hipnozoítos. Dicen Joshi y colaboradores que la resistencia a la PQ ocurre en varios lugares de India y remite a los datos antes citados de esos autores ${ }^{63}$, pero hay que recordar, como insisten Baird y Hoffman, que "las recurrencias después del tratamiento no supervisado no comprueban resistencia", autores estos que afirman que la PQ no permanece universalmente efectiva, aunque no hay evidencia inequívoca ${ }^{9}$.

En esta revisión tampoco se tuvo en cuenta si el tratamiento antimalárico esquizonticida y anti-recurrencias (PQ) fue o no fue supervisado. Se usaron varios esquizonticidas (cloroquina, amodiaquina, artesunato, otros) y todos tuvieron eficacia para curar el ataque agudo, como se exigió para incluir el estudio, pero los estudios no siempre informaron sobre la supervisión a la PQ; esta variable no se tuvo en cuenta para incluir el estudio. La incidencia de recurrencias para los esquemas 210-14 y 210-7 fue $8,16 \%$ y $25,10 \%$; el riesgo relativo $R R$ fue 0,32512. La dosis total fue igual y varió el tiempo de administración (7 y 14 días), pero el mayor tiempo no afectó negativamente la menor incidencia con 210-14. Esto sugiere que en estos estudios la supervisión del tratamiento no parece haber influido, al menos en ese análisis indirecto.

Tampoco se consideró en esta revisión el problema asociado con la calidad de los medicamentos, es decir su origen (fabricante, proveedor final), condiciones de almacenamiento, capacidad antimalárica en el momento de uso, etc. La mala calidad de las drogas antimaláricas existe ${ }^{67}$ y es peor ahora, con la llegada de medicamentos nuevos y más costosos como las artemisininas ${ }^{68-69}$.

En Colombia se reconoce su existencia: "Las estadísticas del Instituto Nacional para la Vigilancia de Alimentos y Medicamentos (Invima) revelan que 4 de cada 10 medicamentos que se ofrecen en el mercado negro son falsificados"70.

No fue objetivo de la revisión medir la frecuencia de eventos adversos a cada esquema de tratamiento con $\mathrm{PQ}$, sino exclusivamente su eficacia anti-recurrencias. Se acepta que muchos de esos eventos son 
dependientes de la dosis y la intensidad de la deficiencia de G6PD; por esto, la PQ debe usarse en sujetos normales según la G6PD, sin embarazo y tomada con algún alimento ${ }^{4,17,65-66,71}$. Una revisión de literatura sobre relaciones entre medicamentos y deficiencia de G6PD acaba de indicar nuevamente que la PQ está entre los "medicamentos que deben evitarse en personas con deficiencia de G6PD"72. Por otra parte, los pacientes sin esta deficiencia tienen excelente tolerancia a altas dosis de PQ y un aspecto adicional para resaltar es la escasa toxicidad imputable a la PQ. La droga al parecer no altera clínicamente las funciones hepática, renal, hematológica ni inmunitaria, pero sí causa metahemoglobinemia, inclusive con la dosis usual, que es muy intensa si existe deficiencia de la enzima glucosa 6 -fosfato deshidrogenasa (G6FD) ligada al cromosoma $X^{9,65-66,73}$.

Conviene recordar, además, que la PQ es útil en malaria para quimioprofilaxis primaria y secundaria y como esquizonticida, aunque por esta última acción poco se emplea $^{9,65-66}$. La PQ tiene actividad in vivo o in vitro contra el hongo Pneumocystis jiroveci, contra varias especies de Leishmania, contra Trypanosoma cruzi, contra virus como Newcastle, contra varias coccidias ${ }^{5,74-75}$. La PQ tiene un rango de aplicaciones potenciales que va de las capacidades anti-recurrencia (P.vivax, $P$.ovale) y gametocitocida $(P$. falciparum) hasta el uso en quimioprofilaxis primaria y como esquizonticida, aunque este último es débil ${ }^{65-66}$.

Tres derivados de la PQ tienen buenas perspectivas como antiparasitarios: la bulaquina o elubaquina y la tafenoquina, como antimaláricos, y la sitamaquina como leishmanicida y agente contra Pneumocystis, ${ }^{5,74-75}$. La tafenoquina también tiene actividad contra Leishmania ${ }^{76}$. Esta droga, cuando se da por vía oral, se absorbe y se metaboliza lentamente, a diferencia de la PQ, por lo que tiene una vida media de 14 días $^{5}$. Pero la tafenoquina no tiene licencia comercial para uso generalizado, también causa hemólisis en sujetos con deficiencia de G6PD ${ }^{77}$, tampoco puede usarse en gestantes ${ }^{75}$, requiere dosis de carga para ser usada en quimioprofilaxis primaria o causal, del orden de 200 $\mathrm{mg} /$ día por 3 días $^{78}$, lo cual es una clara y poderosa desventaja frente a $\mathrm{PQ}^{5,65-66}$.

\section{Referencias}

1. WHO. World Malaria Report 2008. Geneva: WHO; 2008.

2. Baird J, Leksana B, Masbar S, Fryauff D, Sutanihardja M, Suradi L et al. Diagnosis of resistance to chloroquine by Plasmodium vivax: timing of recurrence and whole blood chloroquine levels. Am J Trop Med Hyg 1997; 56: 621-6.

3. Pukrittayakamee S, Vanijanonta S, Chantra A, Clemens $\mathrm{R}$, White N. Blood stage antimalarial efficacy of primaquine in Plasmodium vivax malaria. Infect Dis 1994-a; 169: 932-5.

4. WHO World Health Organization. Guidelines for the treatment of malaria. Documento WHO/HTM/ MAL/2006.1108. Geneve: WHO; 2006.

5. Vale N, Moreira R, Gomes P. Primaquine revisited six decades after its discovery. Eur J Med Chem 2009; 44: 937-53.

6. Edgcomb J, Arnold J, Yount EJ, Alving A, Eichelberger L, Jeffery G et al. Primaquine, SN 13272, a new curative agent in vivax malaria: a preliminary report. J Natl Malar Soc 1950; 9: 285-92.
7. Hill D, Baird J, Parise M, Lewis L, Ryan E, Magill A. Primaquine: report from CDC expert meeting on malaria chemoprophylaxis I. Am J TropMed Hyg 2006; 75: 402-15.

8. Alving A, Arnold J, Hockwald R, Clayman C, Dern R, Beutler E et al. Potentiation of the curative action of primaquine in vivax malaria by quinine and chloroquine. J Lab Clin Med 1955; 46: 301-6.

9. Baird J, Hoffman S. Primaquine therapy for malaria. Clin Infect Dis 2004; 39: 1336-45.

10. Baird J, Rieckmann K. Can primaquine therapy for vivax malaria be improved? Trends Parasitol 2003; 19: 115-20

11. Klein T, Tada M, Lima J, Tang A. Infection of Anopheles darlingi fed on patients infected with Plasmodium vivax before and during treatment with chloroquine plus primaquine in Costa Marques, Rondonia, Brazil. Mem Inst Oswaldo Cruz 1992; 87: 191-5.

12. Duarte E, Pang L, Ribeiro L, Fontes C. Association of subtherapeutic dosages of a standard drug regimen with failures in preventing relapses of vivax malaria. Am J Trop Med Hyg 2001; 65: 471-6. 
13. Alving A, Johnson C, Tarlov A, Brewer G, Kellermeyer R, Carson P. Mitigation of the haemolytic effect of primaquine and enhancement of its action against exoerythrocytic forms of the Chesson strain of Plasmodium vivax by intermittent regimens of drug administration. Bull WHO 1960; 22: 621-31.

14. Clyde D, McCarthy V. Radical cure of chesson strain vivax malaria in man by 7 , not 14, days of treatment with primaquine. Am J Trop Med Hyg 1977; 26: 562-3.

15. Schmidt L, Fradkin R, Vaughan D, Rasco J. Radical cure of infections with Plasmodium cynomolgi: a function of total 8-aminoquinoline dose. Am J Trop Med Hyg 1977; 26(6 pt 1): 1116-28.

16. Baird J. Resistance to therapies for infection by Plasmodium vivax. Clin Microbiol Rev 2009; 22: 508-34. Review.

17. Arnold J, Alving A, Clayman C, Hochwald R. Induced primaquine resistance in vivax malaria. Trans Roy Soc Trop Med Hyg 1961; 55: 345-50.

18. Galappaththy G, Omari A, Tharyan P. Review: Primaquine for preventing relapses in people with Plasmodium vivax malaria. The Cochrane Database of systematic Reviews 2009; Issue 4.

19. Carmona-Fonseca J. Malaria vivax en niños: recurrencias con dosis total estándar de primaquina dada en 3 vs. 7 días. Iatreia 2009-b; 22: 10-20.

20. Primo J. Calculadora para metaanálisis [sitio de internet]. Versión actualizada en 10/7/2009; 1008,00 KB. CASP España. Unidad de Digestivo, Hospital de Sagunto. Disponible en http://www.redcaspe. org/herramientas [Consulta en 1 de julio de 2009]

21. Borenstein M, Rothstin H Comprehensive Meta-Analysis 1.0.25. A computer program for research synthesis. Englewood (NJ, USA): Biostat, 1999.

22. Molinero L. Meta-análisis [sitio de internet]. Asociación de la Sociedad Española de Hipertensión; Liga Epañola para la lucha contra la Hipertensión Arterial. Disponible en http://www.seh-lelha.org/metaanalisis.htm\#TOP [Consulta en 5 de marzo de 2005]

23. Hulley S, Cummings S, Browner W, Grady D, Hearst N, Newman T. Designing Clinical Research. 2 ed. Philadelphia: Lippincott Williams \& Wilkins,2001. p. 14374 .

24. Lozano J, Dennis R El experimento clínico. In: Ruiz A ML (ed). Epidemiología clínica Investigación clínica aplicada. Bogotá: Editorial Panamericana; 2004. p. 23364.

25. Abdon N, Pinto A, da-Silva RS, de-Souza J. Assessment of the response to reduced treatment schemes for vivax malaria. Rev Soc Bras Med Trop 2001; 34: 343-8.

26. Adak T, Sharma V, Orlov V. Studies on the Plasmodium vivax relapse pattern in Delhi, India. Am J Trop Med Hyg 1998; 59: 175-9.
27. Alving A, Hankey D, Coatney G, Jones RJ, Coker W, Garrison P et al. Korean vivax malaria. II. Curative treatment with pamaquine and primaquine. Am J Trop Med Hyg 1953; 6: 970-6.

28. Coatney G, Alving A, Jones RJ, Hankey D, Robinson D, PL. G. et al. Korean vivax malaria. V. Cure of the infection by primaquine administered during long-term latency. Am J Trop Med Hyg 1953; 2: 985-8.

29. Hankey D, Jones JR, Coatney G, Aving A, Coker W, Garrison $\mathrm{P}$ et al. Korean vivax malaria. I. Natural history and response to chloroquine. Am J Trop Med 1953; 2: 958-69.

30. Awab G, Pukrittayakamee S, Imwong M, Dondorp A, Woodrow C, Lee S et al. Dihydroartemisininpiperaquine versus chloroquine to treat vivax malaria in Afghanistan: an open randomized, non-inferiority, trial. Malar J 2010; 9: 105.

31. Boulos M, Amato-Neto V, Dutra A, Di Santi S, Shiroma M. [Frequency of malaria relapse due to P. vivax in a non-endemic region (São Paulo, Brazil)] [Portuguese]. Rev Inst Med Trop Sao Paulo 1991; 33: 143-6.

32. Carmona-Fonseca J, Maestre A. Prevention of Plasmodium vivax malaria recurrence: Efficacy of the standard total dose of primaquine administered over 3 days. Acta Tropica 2009-a; 112: 188-92.

33. Dua V, Sharma V. Plasmodium vivax relapses after 5 days of primaquine treatment, in some industrial complexes of India. Ann Trop Med Parasitol 2001; 95: 655-9.

34. Fairley N. Chemotherapeutic suppression and prophylaxis in malaria. Trans Roy Soc Trop Med Hyg 1945; 38: 311-57.

35. Fernandopulle B, Weeraratne C, Weerasuriya K, Karunaweera N. Efficacy of a five-day course of primaquine in preventing relapses in Plasmodium vivax malaria - a pilot study. Ceylon Med J 2003; 48: 32.

36. Fryauff D, Baird J, Purnomo Awalludin M, Jones T, Subianto B, Richie T, et al. Malaria in a nonimmune population after extended chloroquine or primaquine prophylaxis. Am J Trop Med Hyg 1997; 56: 137-40.

37. Gogtay N, Garg M, Kadam V, Kamtekar K, Kshirsagar N. A 5 days primaquine regimen as anti-relapse therapy for Plasmodium vivax. Trans R Soc Trop Med Hyg 1998; 92: 341. Comment in: Trans R Soc Trop Med Hyg 1998; 1992(1996): 1687.

38. Gogtay N, Desai S, Kadam V, Kamtekar K, Dalvi S, Kshirsagar N. Relapse pattern of Plasmodium vivax in Mumbai: a study of 283 cases of vivax malaria. J Assoc Physicians India 2000; 48: 1085-6. Comment in: J Assoc Physicians India 2002; 1050:1458-99.

39. Hanf M, Stéphani A, Basurko C, Nacher M, Carme B. Determination of the Plasmodium vivax relapse pattern in Camopi, French Guiana. Malar J 2009; 8: 278. 
40. Jelinek T, Nothdurft H, Von Sonnenburg F, Loscher T. Long-term efficacy of primaquine in the treatment of vivax malaria in nonimmune travelers. Am J Trop Med Hyg 1995; 52: 322-4.

41. Leslie T, Rab M, Ahmadzai H, Durrani N, Fayaz M, Kolaczinski J, et al. Compliance with 14-day primaquine therapy for radical cure of vivax malaria: a randomized placebo-controlled trial comparing unsupervised with supervised treatment. Trans R Soc Trop Med Hyg 2004; 98: 168-73.

42. Luxemburger C, van Vugt M, Jonathan S, McGready $\mathrm{R}$, Looareesuwan S, White N, et al. Treatment of vivax malaria on the western border of Thailand. Trans $R$ Soc Trop Med Hyg 1999; 93: 433-8.

43. Maneeboonyang W, Lawpoolsri S, Puangsa-Art S, Yimsamran S, Thanyavanich N, Wuthisen P et al. Directly observed therapy with primaquine to reduce the recurrence rate of plasmodium vivax infection along the Thai-Myanmar border. Southeast Asian J Trop Med Public Health 2011; 42: 9-18.

44. Moon K, Kim Y, Ko D, Park I, Shin D, Kim C. Recurrence rate of vivax malaria in the Republic of Korea. Trans $R$ Soc Trop Med Hyg 2009; 103: 1245-9.

45. Orjuela-Sánchez P, de Santana Filho F, Machado-Lima A, Chehuan Y, Costa M, Alecrim M, et al. Analysis of singlenucleotide polymorphisms in the crt-o and mdr1 genes of Plasmodium vivax among chloroquine-resistant isolates from the Brazilian Amazon region. Antimicrob Agents Chemother 2009; 53: 3561-4.

46. Rowland M, Durrani N. Randomized controlled trials of 5-and 14-days primaquine therapy against relapses of vivax malaria in an Afghan refugee settlement in Pakistan. Trans R SocTrop Med Hyg 1999; 93: 641-3.

47. Schwartz E, Sidi Y. New aspects of malaria imported from Ethiopia. Clin Infect Dis 1998; 26: 1089-91.

48. Schwartz E, Regev-Yochay G, Kurnik D. Short report: a consideration of primaquine dose adjustment for radical cure of Plasmodium vivax malaria. Am J Trop Med Hyg 2000; 62: 393-395.

49. Sharma R, Gautam A, Orlov V, Sharma V. Relapse pattern of Plasmodium vivax in Kheda district, Gujarat. Indian J Malariol 1990; 27: 95-9.

50. Singh N, Mishra A, Sharma V. Radical treatment of vivax malaria in Madhya Pradesh, India. Indian J Malariol 1990; $27: 55-6$.

51. Sinha S, Dua V, Sharma V. Efficacy of 5 day radical treatment of primaquine in Plasmodium vivax cases at the BHEL industrial complex, Hardwar (U.P.). Indian J Malariol 1989; 26: 83-6.

52. Sinha S, Dua V, Sharma V. Malaria relapses and chloroquine resistance at the BHEL industrial complex, Hardwar, India. Trans R Soc Trop Med Hyg 1989; 83: 606.
53. Smoak B, DeFraites R, Magill A, Kain K, Wellde B. Plasmodium vivax infections in U.S. Army troops: failure of primaquine to prevent relapse in studies from Somalia. Am J Trop Med Hyg 1997; 56: 231-4.

54. Solari-Soto L, Soto-Tarazona A, D. M-R, Llanos-Cuentas A. Ensayo clínico del tratamiento de la malaria vivax con esquema acortado de primaquina comparado con el esquema tradicional. Rev Soc Peru Med Interna 2002; 15: $196-9$.

55. Srivastava H, Sharma S, Bhatt R, Sharma V. Studies on Plasmodium vivax relapse pattern in Kheda district, Gujarat. Indian J Malariol 1996; 33: 173-9.

56. Takeuchi R, Lawpoolsri S, Imwong M, Kobayashi J, Kaewkungwal J, Pukrittayakamee S, et al. Directlyobserved therapy (DOT) for the radical 14-day primaquine treatment of Plasmodium vivax malaria on the Thai-Myanmar border. Malar J 2010; 9: 308.

57. Villalobos-Salcedo J, Tada M, Kimura E, Menezes M, Pereira da Silva L. In-vivo sensitivity of Plasmodium vivax isolates from Rondonia (western Amazon region, Brazil) to regimens including chloroquine and primaquine. Ann Trop Med Parasitol 2000; 94: 749-58.

58. da Silva R, Pinto A, Calvosa V, de Souza J. Esquemas terapêuticos encurtados para o tratamento de malária por Plasmodium vivax. Short course schemes for vivax malaria treatment. Rev Soc Bras Med Trop 2003; 36: 2359.

59. Pinto A, Ventura A, Calvosa V, Silva-Filho M, Santos M, Silva R, et al. Eficácia de quatro esquemas terapêuticos para malaria vivax em crianças. J Pediatr 1998; 74: 222-7.

60. Goller J, Jolley D, Ringwald P, Biggs B. Regional differences in the response of plasmodium vivax malaria to primaquine as anti-relapse therapy. Am J Trop Med Hyg 2007; 76: 203-7.

61. Kshirsagar N. Malaria: anti malarial resistance and policy ramifications and challenges. J Postgrad Med 2006; 52: 291-3.

62. Garnham P. Hypnozoites and "relapses" in Plasmodium vivax and in vivax-like malaria. Trop Geogr Med 1988; 40: 187-95.

63. Joshi H, Prajapati S, Verma A, Kang'a S, Carlton J. Plasmodium vivax in India. Trends Parasitol 2008; 24: 228-35.

64. Santos J, Luz F, Deckers F, Tauil P. Subdoses of primaquine in overweight patients and malaria vivax relapses: report of two cases in the Federal District, Brazil. Rev Soc Bras Med Trop 2010; 43: 749-50.

65. Carmona-Fonseca J. Profilaxis primaria con primaria para el paludismo. Revisión. Anuario de Enfermedades Infecciosas (Medellín) 2005; 2: 51-84.

66. Carmona-Fonseca J. La primaquina tiene alta eficacia en la quimioprofilaxis primaria simple antipalúdica. Metanálisis. Iatreia 2006; 19: 244-60. 
67. Nogueira F, Moreira-Campos L, Santos R, Pianetti G. Quality of essential drugs in tropical countries: evaluation of antimalarial drugs in the Brazilian Health System. Rev Soc Bras Med Trop 2011; 44: 582-6.

68. Newton P, Fernandez F, Plancon A, Mildenhall D, Green $\mathrm{M}$, al e. A collaborative epidemiological investigation into the criminal fake artesunate trade in South East Asia. PLoS Med 2008; 5(2): e32.

69. Bate R, Coticelli P, Tren R, Attaran A. Antimalarial drug quality in the most severely malarious parts of Africa - A six country study. PLoS ONE 2008; 3(5):e2132.

70. Arias F. Convenio con Invima contra el tráfico de medicamentos en Colombia. El Colombiano (periódico) Medellín, Colombia, 9 de noviembre de 2004.

71. Ferreira M, Gomes MdS, Vieira J. [Methemoglobinemia in patients with Plasmodium vivax receiving oral therapy with primaquine] [en Portugués]. Rev Soc Bras Med Trop 2011; 44: 113-5.

72. Youngster I, Arcavi L, Schechmaster R, Akayzen Y, Popliski H, Shimonov J et al. Medications and glucose6-phosphate dehydrogenase deficiency: an evidencebased review. Drug Saf 2010; 33: 713-26.

73. Carmona-Fonseca J, Alvarez G, Maestre A. Methemoglobinemia and adverse events in Plasmodium vivax malaria patients associated with high doses of primaquine treatment. Am J Trop Med Hyg 2009-c; 80: 188-93.
74. Tekwani B, Walker L. 8-Aminoquinolines: future role as antiprotozoal drugs. Curr Opin Infect Dis 2006; 19: 62331.

75. Crockett M, Kain K. Crockett M, Kain KC. Tafenoquine: a promising new antimalarial agent. Expert Opin Investig Drugs. 2007; 16(5):705-15. Expert Opin Investig Drugs 2007; 16: 705-15.

76. Manzano J, Carvalho L, García-Hernández R, Poveda J, Ferragut J, Castanys S, et al. Uptake of the antileishmania drug tafenoquine follows a steroldependent diffusion process in Leishmania. $J$ Antimicrob Chemother 2011; 66: 2562-5.

77. Shiraki H, Kozar M, Melendez V, Hudson T, Ohrt C, Magill A, et al. Antimalarial activity of novel 5-aryl-8aminoquinoline derivatives. J Med Chem 2011; 54: 13142.

78. Charles B, Miller A, Nasveld P, Reid M, Harris I, Edstein M. Population pharmacokinetics of tafenoquine during malaria prophylaxis in healthy subjects. Antimicrob Agents Chemother 2007; 51: 2709-15.

Recebido em: 09/06/11 Versão final apresentada em: 23/01/12 Aprovado em: 10/02/12 